\title{
Conservative treatment for Insertional Achilles Tendinopathy: platelet-rich plasma and focused shock waves. A retrospective study
}

\author{
Davide Erroi ${ }^{1}$ \\ Matilde Sigona ${ }^{1}$ \\ Tania Suarez ${ }^{1}$ \\ Donatella Trischitta ${ }^{1}$ \\ Antonio Pavan² \\ Maria Chiara Vulpiani ${ }^{1}$ \\ Mario Vetrano ${ }^{1}$ \\ 1 Physical Medicine and Rehabilitation Unit, \\ Sant'Andrea Hospital, "Sapienza" University \\ of Rome, Italy \\ 2 Department of Immunohematology and Transfu- \\ sion, Sant'Andrea Hospital, "Sapienza" \\ University of Rome, Italy
}

Corresponding author:

Mario Vetrano

Physical Medicine and Rehabilitation Unit

Sant'Andrea Hospital, "Sapienza" University

of Rome

Via di Grottarossa 1035-1039

00189 Roma, Italy

E-mail: mariovetrano@gmail.com

\section{Summary}

Background: Insertional Achilles tendinopathy (IAT) represents a serious challenge for both physiatrists and surgeons. Here we analyse the results obtained by two conservative treatments [platelet-rich plasma (PRP) injections and focused extracorporeal shock-wave therapy (ESWT)] in physically active patients with IAT.

Methods: During two consecutive periods, 45 consecutive patients with IAT were treated with 3 sessions of ESWT (2400 impulses at 0.17-0.25 $\mathrm{mJ} / \mathrm{mm}^{2}$ per session) (24 cases between September 2011 and July 2013) or with 2 autologous PRP injections over two weeks ( 21 cases between September 2013 and July 2015). All patients were evaluated at 0, 2-, 4-, 6-month follow-up after therapy. The outcome measures were VISA-A, VAS, Patient Satisfaction.

Results: Intra-group analysis showed a significant improvement of VISA-A and VAS scores in both groups at all time-points. No differences between groups were observed for VAS and VISA-A scores at all time-points, excepted for VISA-A at 4-months in favour of ESWT group $(P=0.049)$. $P a-$ tient satisfaction increased progressively $(>70 \%$ at 6 months) and with no differences between two groups.

Conclusion: Both ESWT and PRP therapy are effective and safe. Our study confirms the success of these conservative treatments in Achilles tendinopathy, even in the insertional one. Level of evidence: Illa.

KEY WORDS: foot pain, conservative treatment, physical therapy, sport rehabilitation.

\section{Introduction}

Achilles tendinopathy is the most common overuse syndromes of more or less physically active people ${ }^{1}$. Its pathogenesis remains unclear; actually this tendinopathy seems to be a multifactorial condition, to which intrinsic and extrinsic factors concur ${ }^{2}$. It occurs when repetitive exercise of the Achilles tendon overcomes its physiological threshold leading to inflammation of its sheath, degeneration of its body, or a combination of both ${ }^{3}$. Clinical features of Achilles tendinopathy are tendon pain, swelling, and impaired performance ${ }^{4-6}$. There are many classifications of Achilles tendinopathy according to clinical, histological, and anatomical criteria ${ }^{7-9}$. In particular, main clinical classification distinguishes a non-insertional tendinopathy (NAT) and insertional tendinopathy (IAT). According to several research studies, the first one occurs in $55-65 \%$ of cases and the second one in the $20-25 \%^{10-17}$. IAT is more common in the physically active persons, whereas NAT is diagnosed more often in older, less athletic, and overweight persons ${ }^{18,19}$.

Despite high frequency of IAT in young active people and despite its impact on work and social life, aetiology and pathogenesis of this disease have not been clearly defined yet. Therefore, absence of a clear cause-effect association makes treatment of IAT difficult. It is hypothesized that the pathology is similar in both mid-tendon and insertional tendinopathy: a degeneration of the tendon matrix with loss of collagen fibre structure as a failed healing response $20-23$. However, treatments of Achilles tendinopathy are less effective in IAT that in NAT, as if insertional Achilles tendon represents a separate entity, such as an "enthesis organ" 24,25 , constituted by tendon, bursae, bone and fat pad. 
Conservative treatment for Insertional Achilles Tendinopathy: platelet-rich plasma and focused shock waves. A retrospective study

The first treatment of Achilles tendinopathy is conservative and is usually similar for IAT e NAT. Many treatments have been proposed, including rest, antiinflammatory medications, physical therapy, orthosis and immobilization. This approach has proven to be relativity effective in the majority of cases $^{26-36}$. Among non-surgical treatments for Achilles tendinopathy there are the extracorporeal shock-wave therapy (ESWT) and platelet-rich plasma (PRP) injections. The effectiveness of ESWT and PRP in the treatment of Achilles tendinopathy was reported in previous studies $^{36-43}$. Both are regenerative treatments but their mechanism of action in vivo is still unclear. The rational of applying in vivo ESWT on tendons is supported by the results obtained with shockwaves on models in vitro, such as enhancing cell proliferation, migration and secretory activity of human primary cultured tenocytes ${ }^{44}$. Basic science studies about PRP mechanism action show that growth factors in PRP significantly enhance the healing of tendon injuries such as tendinopathy 45 .

In scientific literature, there is no evidence about the most effective non-surgical intervention for IAT ${ }^{46}$.

The aim of this retrospective study is to analyse the results obtained by two treatments (ESWT and PRP) in physically active patients with chronic IAT focusing on effectiveness, pain reduction, functional recovery and satisfaction in time span of six months after end of the treatments.

\section{Materials and methods}

This work is a retrospective study involving 45 homogeneous consecutive patients affected by IAT, who were examined and treated in the Physical Medicine and Rehabilitation Unit of Sant'Andrea Hospital of Rome ("Sapienza" University of Rome). They are people who referred to our ambulatory for Achilles disorders in the period from September 2011 to July 2015 and that received diagnosis of IAT by the same clinician. During the evaluation phase, each patient performed a clinical examination, an ultrasound (US) examination using a sonographic unit (Technos MP, Esaote ${ }^{\circledR}$, Genova, Italy) with a multi-frequency linear array probe $(7,5-12 \mathrm{MHz})$, made by a clinician with ten years' experience in musculoskeletal US, and a radiographs of the calcaneus to identify tendon calcification. IAT was defined as the localized pain at insertion of the Achilles tendon into calcaneus, strong tenderness to pressure and a limitation of physical function.

Inclusion criteria of this study were: evidence of thickening with focal area of hypoechoic change and of fibril discontinuity at US examination; a diagnosis of unilateral chronic insertional Achilles tendinopathy for at least six months before treatment; refractory to traditional conservative treatment; a washout period of twelve weeks from any non-operative therapy; age range from 20 to 70 years; ability to complete questionnaires; regular physical activity or agonistic sports. Patients were included even if retrocalcaneal bursitis, shown at US examination, coexisted with IAT. Exclusion criteria were: bilateral diagnosis; signs or symptoms of other coexisting foot lesions; midportion Achilles tendinopathy; evidence of Haglund deformity at radiographs; congenital or acquired deformities of the knee and ankle; foot surgery or injection therapy with corticosteroids in the past 3 months; systemic disorders such as diabetes, rheumatoid arthritis, coagulopathies, infections, immunodepression, or neoplastic diseases; therapy with anticoagulants-antiaggregants; platelet values of fewer than $150,000 / \mathrm{mm}^{3}$; pregnancy.

Having reached an accurate diagnosis of IAT, we submitted these 45 patients to two conservative treatments in two consecutive periods, subject to this retrospective study. During the first period, between September 2011 and July 2013, 24 patients were treated with ESWT (ESWT Group); in the latter, between September 2013 and July 2015, 21 patients were undergone to PRP injections (PRP Group). Informed consent for both treatments was obtained by all patients ${ }^{47}$.

\section{Methods of treatment}

\section{PRP injections}

Platelet-rich plasma gel (PRP) was supplied by Immunohematology and Transfusion Unit (SIMT) of the Hospital. All 23 patients in this group received two autologous pure PRP injections over two weeks (1 injection per week for two weeks) under ultrasound guidance. All treatments were performed by the same trained Author who was not involved in assessing the patients before or after the treatment. Ten $\mathrm{ml}$ of blood were collected from the patient using RegenKit ${ }^{\circledR}$ BCT-3 (Regenlab®, Le Mont-sur-Lausanne, Switzerland), whose technology removed more than $96 \%$ of the granulocytes. The tube was then centrifuged for 5 minutes at $1500 \mathrm{~g} \mathrm{(1300} \mathrm{to} 1500 \mathrm{rpm})$. Centrifugation resulted in 6-7 $\mathrm{ml}$ of PRP. In order to obtain a higher cellular concentration, before proceeding to the platelet re-suspension we delicately removed $2 \mathrm{ml}$ of the upper layer of the platelet-poor supernatant (PPP). The remaining $2 \mathrm{ml}$ of cellular deposit resulted in a mean platelets concentration of $0.89-1.1 \times 109$ $\mathrm{ml}$ (approximately 3 - 5 times baseline concentration). The designated injection location was recorded before the injection (hypoechogenicity of the tendon). The injection technique involved a single skin portal using a 22-g needle and then multiple small aliquots into the tendinous lesion, with colour Doppler guidance. Approximately $2 \mathrm{ml}$ of pure PRP was injected and no local anaesthesia was applied. After the injections, the patient rested in a supine position without moving the leg for 15 minutes and moderate compression bandage was applied with indication to remove it at the end of the day. Patients were allowed full loading of the limb immediately and could perform normal activities of daily living. If necessary, patients were allowed to use acetaminophen, but the use of nonsteroidal anti-inflammatory medication was prohibited. 


\section{ESWT}

Patients, treated in the first period, received three sessions of focused ESWT by the same expert clinician. Each session consisted of 2400 impulses administered with an energy flux density ranged from 0.17 to $0.25 \mathrm{~mJ} / \mathrm{mm}^{2}$. Intensity was dictated by patient tolerance. The sessions were administered at weekly intervals. We used a focused electromagnetic shock wave device (Modulith ${ }^{\circledR}$ SLK, STORZ Medical, Switzerland). The procedure was performed with the patient in prone position. The treatment area was prepared with a coupling ultrasound gel to minimize the loss of shock wave energy at the interface between applicator tip and skin. Inline ultrasonic guidance was used to focus the shock waves on the injury area in the insertional Achilles tendon. No local anaesthesia was applied.

\section{Post-treatment protocol}

After last treatment session with PRP injections or with ESWT, patients were given an identical home exercise protocol for Achilles tendinopathy, to perform daily for eight weeks until the first follow-up time point and twice a week for four following weeks. It was explained by the clinician and was carried out without physiotherapist supervision. It consisted of five phases; 1) initial triceps surae stretching with progressive intensity ( 30 seconds $\times 2$ series on both sides); 2) triceps surae and Achilles tendon eccentric training avoiding the ankle dorsiflexion ${ }^{26}$ (15 repetitions at extended knee $\times 3$ series, 15 repetitions at flexed knee $\times 3$ series); 3) foot proprioceptive exercises; 4) final triceps surae stretching (30 seconds $\times 2$ series on both sides); 5) application of ice (10 minutes) on the affected area after completion of the exercise session. Four weeks after treatment patients could return gradually to previous training activities if there was minimal or no pain; whereas complete return to sports activities took place in accordance with the patient's pain tolerance and absence of clinical signs.

\section{Outcome assessments}

We assessed patients before treatment and at three follow-up time points: 2, 4, and 6 months after end of treatment. During each evaluation, three parameters were noted: Victorian Institute of Sports AssessmentAchilles (VISA-A) score, Visual Analog Scale (VAS), and patient satisfaction according to the Roles and Maudsley score.

\section{VISA-A score}

At each visit, patients were requested to complete the Italian version of the Victorian Institute of Sports Assessment-Achilles (VISA-A) questionnaire, which was designed specifically for patients suffering from Achilles tendinopathy to assess severity of symptoms, function, and ability to participate in sport. VISA-A is the only published clinical scale validated for Achilles tendinopathy. The questionnaire contains eight questions covering the 3 domains of pain (questions 1-3), function (questions 4-6), and sport activity (questions 7 and 8 ). Questions 1 through 7 are marked out of 10 , while question 8 carries a maximum of 30 . Scores are summed up to give a total out of 100 . For question 8 , participants must answer only part $\mathrm{A}, \mathrm{B}$, or $\mathrm{C}$. The maximum score possible, which corresponds to an asymptomatic athlete, is 100 points. The theoretical minimum is 0 points 48,49 .

\section{VAS}

Self-reported levels of pain were recorded on the 10$\mathrm{cm}$ horizontal scale VAS, with $0 \mathrm{~cm}$ corresponding to "no pain" and $10 \mathrm{~cm}$ corresponding to "the worst pain possible". The VAS scale has proved a reliable and sensitive scale for pain and has been widely used in previous clinical studies ${ }^{50}$.

\section{Patient satisfaction}

The Roles and Maudsley score (RMS) is a 4-point subjective patient assessment of pain and limitations of activity. On this scale, 1 point is defined as an "Excellent" result (no pain, full movement and full activity), 2 points are defined as a "Good" result (occasional discomfort, full movement and full activity), 3 points are defined as a "Fair" result (some discomfort after prolonged activity), and 4 points indicates a "Poor" result (symptoms identical or worse than pre-treatment condition). We defined a RMS grade of good or excellent as "satisfactory" results, whereas fair or poor indicated "unsatisfactory" results ${ }^{51}$.

\section{Statistical analysis}

Continuous variables were presented as mean and standard deviation, while categorical variables were described by frequency and percentage. Analysis of variance (ANOVA) was used for within-group and between-group analyses of parametric variables at each assessment time points. A binomial logistic regression was performed to determine the effects of the VISA-A improvement from the baseline value over time (independent variables) on the likelihood that patients have satisfactory results at 6-month followup time point (dependent variable). The $z$ test for proportions was used to compare the percentages of satisfactory results in both groups. Significant differences were assumed at $\mathrm{P}<05$. All analyses were performed with the software IBM SPSS for MAC v. 22 (IBM SPSS Inc., Chicago, IL).

\section{Results}

Patients of the two treatment groups appeared homogeneous in term of pre-treatment characteristics (age, sex, level of sports participation, and pre-treatment clinical status) (Tab. I). All patients were assessed at the scheduled follow-up time points and no patient was undergone to surgery during this period.

No clinically relevant side effects were detected in both groups. Only five patients in the PRP injections group complained local pain and discomfort that occurred soon after injection and gradually disappeared. In the ESWT group, transient reddening of 
Conservative treatment for Insertional Achilles Tendinopathy: platelet-rich plasma and focused shock waves. A retrospective study

Table I. Baseline characteristics of both groups.

\begin{tabular}{|c|c|c|c|}
\hline Characteristic & PRP Group & ESWT Group & $P$ Value \\
\hline Patients, no. & 21 & 24 & \\
\hline Age, mean (SD), $y$ & $47.7(9.3)$ & $53.2(13.1)$ & 0.111 \\
\hline Gender, no. (\%) & & & 0.546 \\
\hline Men & $13(61.9)$ & $17(70.8)$ & \\
\hline Women & $8(38.1)$ & $7(29.2)$ & \\
\hline Affected Achilles Tendon, no. (\%) & & & 0.376 \\
\hline Right & $10(47.6)$ & $15(62.5)$ & \\
\hline Left & $11(52.4)$ & $9(37.5)$ & \\
\hline Tendon Calcifications, no. (\%) & & & 0.543 \\
\hline Yes & 7 (33.3) & $11(45.8)$ & \\
\hline No & $14(66.7)$ & $13(54.2)$ & \\
\hline Retrocalcaneal Bursitis, no. (\%) & & & 0.936 \\
\hline Yes & $9(42.8)$ & $10(41.7)$ & \\
\hline No & $12(57.2)$ & $14(58.3)$ & \\
\hline Duration of symptoms, mean (SD), mo. & $14.3(6.9)$ & $13.7(5.4)$ & 0.756 \\
\hline Sport activity, no. (\%) & & & 0.442 \\
\hline Elite athletes & $5(23.8)$ & $3(12.5)$ & \\
\hline Non elite athletes & 16 (76.2) & $21(87.5)$ & \\
\hline
\end{tabular}

Abbreviations: PRP, platelet-rich plasma; ESWT, extracorporeal shock wave therapy; no., number; SD, standard deviation.

the skin occurred after treatment, but no bruising was seen.

\section{VISA-A score}

The two treatments were effective in increasing the baseline VISA-A score values at 2-, 4-, and 6-month follow-up $(\mathrm{P}<.005$ at each follow-up time point). The VISA-A score in the PRP injections group showed significant progressive improvement at all points of follow-up. In the ESWT group, there was gradual improvement during follow-up, except for the 4 and 6month controls.

Between-groups analysis showed that the VISA-A score has no significant difference between two treatments during two evaluations: before treatments (PRP injections group, $52.8 \pm 14.2$; ESWT group, $50.6 \pm 14.8 ; P=0.624)$ and at the 2-month follow-up (PRP injections group, $66.6 \pm 17.2$; ESWT group, $67.0 \pm 17.5 ; P=0.939)$. Differently, the ESWT group showed significantly better improvement than PRP group at 4-month follow-up (PRP injections group, $72.4 \pm 19.5$; ESWT group, $82.8 \pm 15.2 ; \mathrm{P}=0.049$ ). No significant difference between the groups is noted at 6-month follow-up time point (PRP injections group, $82.0 \pm 18.1$; ESWT group, $86.5 \pm 14.4$; $\mathrm{P}=$ 0.368) (Tab. II).

\section{VAS}

Both treatments reduced significantly the baseline VAS values at 2-,4-, and 6-month follow-up (all P values $<.005)$.
The VAS score showed no significant difference between two groups at any assessments: before treatment (PRP injections group, $5.9 \pm 1.0$; ESWT group, $6.4 \pm 1.3 ; \mathrm{P}=0.153)$; at the 2-month follow-up (PRP injections group, $3.4 \pm 2.1$; ESWT group, $3.5 \pm 2.0$; $\mathrm{P}$ $=0.855$ ); at 4-month follow-up (PRP injections group, $3.0 \pm 1.9$; ESWT group, $2.5 \pm 2.3 ; P=0.442)$; at $6-$ month follow-up (PRP injections group, $2.6 \pm 1.9$; ESWT group, $1.5 \pm 2.1 ; P=0.078$ ) (Tab. III).

ANOVA for repeated measures showed a positive time effect, but not a treatment or intercept effect.

\section{Patient satisfaction}

Patient satisfaction increased progressively in both treatments at every follow-up time point. There was no significant difference between ESWT group and PRP injections group of satisfaction reported during assessment at 2-, 4-, 6-month follow-up (Tab. IV). Considering the entire sample of 45 subjects, $40.9 \%$ of satisfied patients at 2-month, $56.2 \%$ at 4 -month, and $77.8 \%$ at 6 -month follow-up have an improvement $>30$ points of VISA-A score (Tab. V).

The binomial logistic regression model was statistically significant $P<.001$. The model was able to explain $66.1 \%$ of the variance in patient satisfaction and correctly classified $86.7 \%$ of cases. Sensitivity was $55.6 \%$, specificity was $94.4 \%$, positive predictive value was $94.7 \%$ and negative predictive value was $71.4 \%$. Of the three predictor variables only 6 -month VISA-A improvement was statistically significant. Specifically, the increasing VISA-A at 6-month follow- 


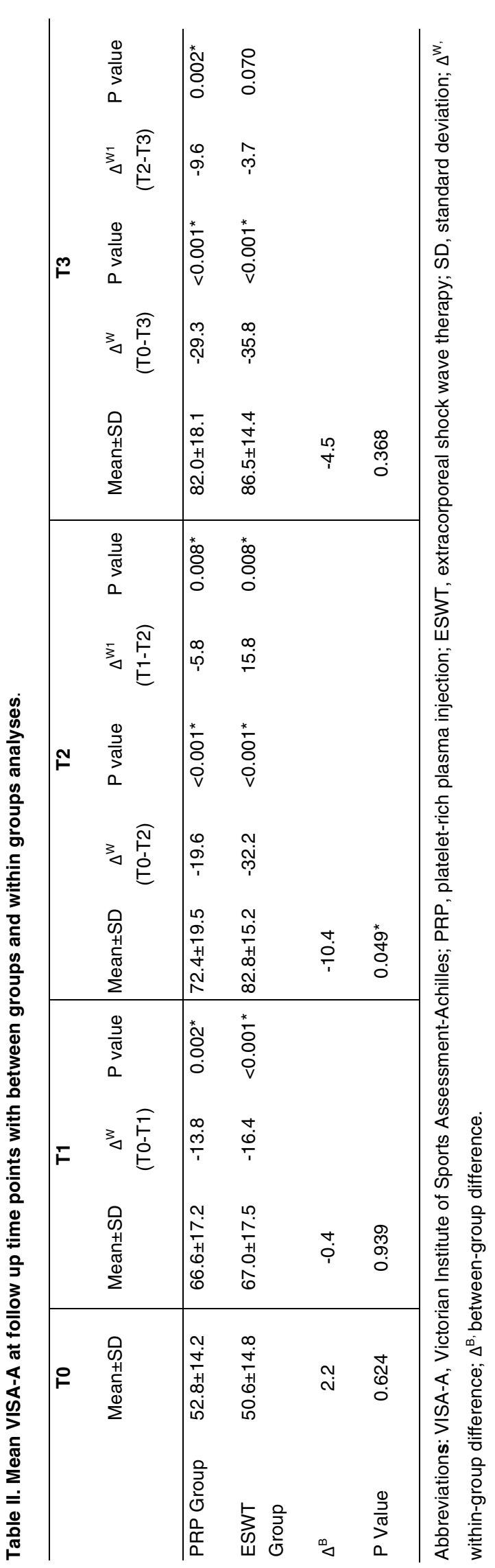

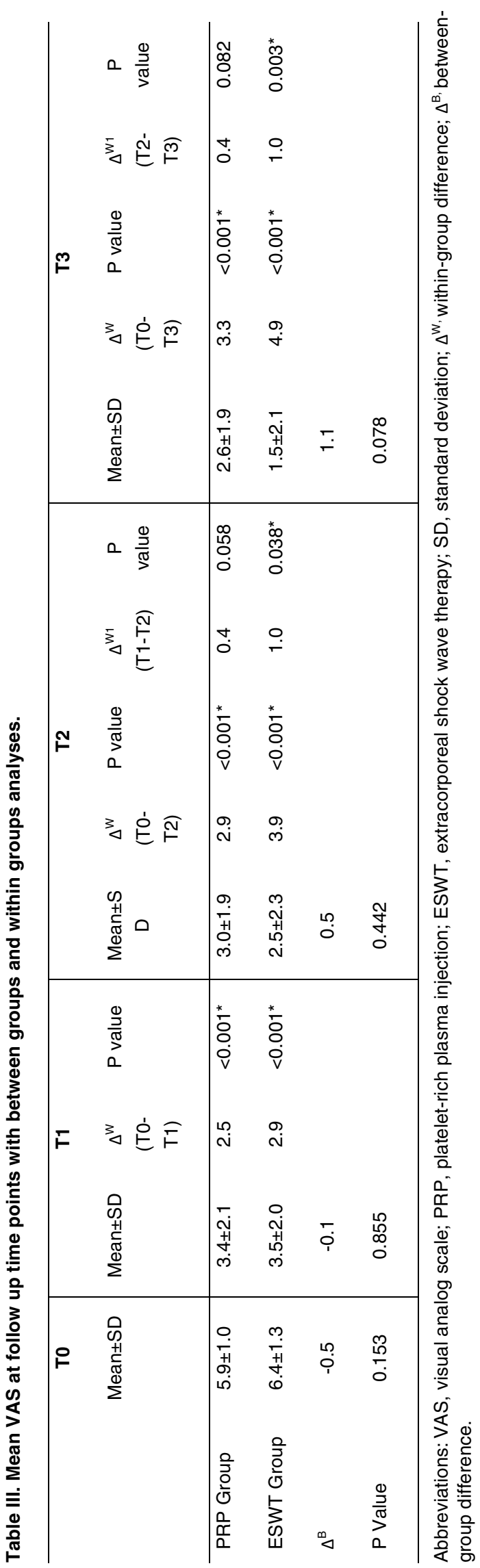

Muscles, Ligaments and Tendons Journal 2017;7 (1):98-106 
Conservative treatment for Insertional Achilles Tendinopathy: platelet-rich plasma and focused shock waves. A retrospective study

Table IV. Patient satisfaction at 2-, 4-, and 6-month follow-up.

\begin{tabular}{|c|c|c|c|}
\hline Results, no. (\%) & PRP Injection Group (no.=21) & ESWT Group (no.=24) & $P$ Value \\
\hline 2-month follow-up & & & 0.768 \\
\hline Satisfactory & $11(52,4)$ & $11(45,8)$ & \\
\hline Unsatisfactory & $10(47,6)$ & $13(54,2)$ & \\
\hline 4-month follow-up & & & 0.323 \\
\hline Satisfactory & $13(61,9)$ & $19(79,2)$ & \\
\hline Unsatisfactory & $8(38,1)$ & $5(20,8)$ & \\
\hline 6-month follow-up & & & 0.267 \\
\hline Satisfactory & $15(71,4)$ & $21(87,5)$ & \\
\hline Unsatisfactory & $6(28,6)$ & $3(12,5)$ & \\
\hline
\end{tabular}

Abbreviations: no., numbers; PRP, platelet-rich plasma; ESWT, extracorporeal shock wave therapy.

Table V. Satisfaction in relation to VISA-A improvement classes at 2-, 4- and 6-month follow-up.

\begin{tabular}{|c|c|c|c|}
\hline Follow-up & VISA-A improvement & Satisfactory, no. (\%) & Unsatisfactory, no. (\%) \\
\hline \multirow[t]{6}{*}{ 2-month } & & $22(48.9)$ & $23(51.1)$ \\
\hline & $\Delta \mathrm{VISA}-\mathrm{A}>30 \mathrm{pts}$ & $9(40.9)$ & - \\
\hline & $\Delta$ VISA-A $20-30$ pts & $4(18.2)$ & $4(17.4)$ \\
\hline & $\Delta$ VISA-A $10-20$ pts & $5(22.7)$ & $4(17.4)$ \\
\hline & $\Delta \mathrm{VISA}-\mathrm{A} \quad 0-10 \mathrm{pts}$ & $3(13.7)$ & $12(52.2)$ \\
\hline & $\Delta \mathrm{VISA}-\mathrm{A} \leq 0 \mathrm{pts}$ & $1(4.5)$ & $3(13)$ \\
\hline \multirow[t]{6}{*}{ 4-month } & & $32(71.1)$ & $13(28.9)$ \\
\hline & $\Delta \mathrm{VISA}-\mathrm{A}>30 \mathrm{pts}$ & $18(56.2)$ & - \\
\hline & $\Delta$ VISA-A $20-30$ pts & $8(34.4)$ & $5(38.5)$ \\
\hline & $\Delta$ VISA-A $10-20$ pts & $3(9.4)$ & $1(7.7)$ \\
\hline & $\Delta$ VISA-A 0-10 pts & - & $2(15.3)$ \\
\hline & $\Delta \mathrm{VISA}-\mathrm{A} \leq 0 \mathrm{pts}$ & - & $5(38.5)$ \\
\hline \multirow[t]{6}{*}{ 6-month } & & $36(80)$ & $9(20)$ \\
\hline & $\Delta \mathrm{VISA}-\mathrm{A}>30 \mathrm{pts}$ & $28(77.8)$ & $2(22.2)$ \\
\hline & $\Delta$ VISA-A $20-30$ pts & $6(16.6)$ & $1(11.1)$ \\
\hline & $\Delta$ VISA-A $10-20$ pts & $1(2.8)$ & $1(11.1)$ \\
\hline & $\Delta$ VISA-A 0-10 pts & $1(2.8)$ & $4(44.5)$ \\
\hline & $\Delta \mathrm{VISA}-\mathrm{A} \leq 0 \mathrm{pts}$ & - & $1(11.1)$ \\
\hline
\end{tabular}

Abbreviations: no., numbers; $\triangle$ VISA-A, variation of Victorian Institute of Sports Assessment-Achilles; pts, points.

up was associated with an increased likelihood of obtaining satisfactory results, with an odds ratio of 0.869 for each increase in one unit of this variable (Tab. VI).

\section{Discussion}

Results analysis of our retrospective study shows a beneficial effect of ESWT and PRP injections on pain reduction and recovery of physical activity of patients during the 6-month follow-up period. Many research studies and a review article published in $2013^{46}$ support the role of ESWT for treatment of IAT, whereas at our knowledge there is no evidence in the literature about the use of PRP injections for this tendinopathy. In 2006, Furia ${ }^{36}$ published a case control study about the effectiveness of ESWT on IAT. Thirty-five subjects were treated with ESWT (3000 shocks in one session; $0.21 \mathrm{~mJ} / \mathrm{mm}^{2}$; total energy flux density: 604 $\mathrm{mJ} / \mathrm{mm}^{2}$ ), and 33 with non-operative therapy (control group). All ESWT procedures were performed using a 
Table VI. Logistic regression predicting likelihood of patient satisfaction based on VISA-A improvement over time.

\begin{tabular}{|c|c|c|c|c|c|c|c|c|}
\hline & \multirow{2}{*}{ B } & \multirow{2}{*}{ SE } & \multirow{2}{*}{ Wald } & \multirow{2}{*}{$d f$} & \multirow{2}{*}{$\mathbf{P}$} & \multirow{2}{*}{$\begin{array}{l}\text { Odds } \\
\text { Ratio }\end{array}$} & \multicolumn{2}{|c|}{$95 \% \mathrm{Cl}$ for Odds Ratio } \\
\hline & & & & & & & Lower & Upper \\
\hline \multicolumn{9}{|l|}{ VISA-A improvement } \\
\hline 2-month follow-up & .132 & .076 & 3.048 & 1 & .081 & 1.141 & .984 & 1.324 \\
\hline 4-month follow-up & -.136 & .075 & 3.247 & 1 & .072 & .873 & .753 & 1.012 \\
\hline 6-month follow-up & -.140 & .052 & 7.517 & 1 & $.007^{\star}$ & .869 & .784 & .963 \\
\hline
\end{tabular}

local anaesthesia field block or a nonlocal anaesthesia. He concluded that ESWT was an effective treatment for this tendinopathy with beneficial effects at follow-up at 12 months and that local field block anaesthesia may have decreased the effects of this therapy.

In a randomized controlled trial, Rompe et al. ${ }^{39} \mathrm{com}$ pared the effect of two treatments in 50 patients with chronic recalcitrant IAT, eccentric training or ESWT (2000 pulses $\times 3$ sessions; flux density: 0.12 $\mathrm{mJ} / \mathrm{mm}^{2}$ ). The Authors demonstrated that the probability for recovery was significantly lower after eccentric loading than ESWT. We note that the VISA-A score at 4-month in ESWT group, reported by Rompe, is $79.4 \pm 10.4$. In our sample, a similar mean score at 4-month follow-up was obtained in ESWT group $(82.8 \pm 15.2)$ and it was significantly greater in comparison with that obtained PRP injections group $(72.4 \pm 19.5)$

Vulpiani et al. ${ }^{38}$ described the effects of ESWT in a long-term follow-up observational study of patients affected by Achilles tendinopathy, without discerning IAT from NAT. One hundred-five patients (127 tendons) were treated with focused ESWT (average four sessions of ESWT, in each session 1500-2500 with an energy varying between 0.08 and $0.40 \mathrm{~mJ} / \mathrm{mm}^{2}$ ). These Authors found persistent positive effect after 13-24 months.

In 2014, in a prospective clinical trial, Notarnicola et al. ${ }^{21}$ evaluated the effectiveness of a flow of Cold air and High Energy Laser Therapy (CHELT) versus ESWT in 60 subjects with chronic IAT. ESWT group, received three sessions at 3- to 4-day intervals of 1600 impulses with an energy flux density of 0.05 $0.07 \mathrm{~mJ} / \mathrm{mm}^{2}$. After treatment both groups performed stretching and eccentric exercise for 2 months. Results of CHELT group were better than ESWT group. Moreover, they hypothesized that CHELT therapy could also be useful in treating acute tendinopathies with more inflammation, while the ESWT should be better in the treatment of more chronic cases, which are more likely to get worse or not respond to treatment, as highlighted in the NICE guidelines ${ }^{37}$.
In a recent study, Taylor et al. ${ }^{52}$ investigated the longterm response of a cohort of patients with refractory Achilles tendinopathy undergoing ESWT according to the guidelines issued by NICE. Forty-six patients were treated with radial shockwaves (2500 pulses $\times 3$ sessions at weekly intervals, pressure until 2.5 Bar). VAS and VISA-A scores were subsequently obtained at 6,16 weeks and 2 years post treatment. Likert satisfaction scores were also obtained at these follow-up points. Their study revealed an improvement in patient symptoms of both NAT and IAT following treatment with ESWT, besides they supposed a possibility that associated bony abnormalities in patients with IAT had an adverse effect on the efficacy of ESWT. At our knowledge, research studies about PRP injections for IAT treatment are absent and only a few studies focused on PRP injections for unspecified Achilles tendinopathy. In 2012, Ferrero et al. ${ }^{42}$ conducted a prospective study that evaluated the effectiveness of ultrasound (US)-guided intratendinous administration of PRP to treat jumper's knee and Achilles tendinopathy in a population practiced sports at the competitive or amateur level. The patients in his study were a total of 28 patellar tendons in 24 patients and 30 Achilles tendons in 24 patients. Also in this study there was not distinction between IAT and NAT. Their conclusion was that PRP injection in Achilles tendinopathy resulted in a significant and lasting improvement of clinical symptoms and led to recovery of the tendon matrix potentially helping to prevent degenerative lesions.

In 2015, a systematic review studied the available clinical evidence concerning the application of PRP in the treatment of patellar and Achilles tendinopathy. It included only trials about NAT. This review asserted that, although the clinical data available were not univocal, PRP is an option for the management of Achilles tendinopathy and a second-line approach for tendinopathies not responsive ${ }^{53}$.

Recently two reviews investigated about effectiveness of PRP treatments in tendon-related disorders. Filardo et al. ${ }^{54}$ analysed twenty papers that described PRP injections as a conservative management for 
Conservative treatment for Insertional Achilles Tendinopathy: platelet-rich plasma and focused shock waves. A retrospective study

chronic Achilles tendinopathy. He concluded that, according to current evidence, the application of PRP is not justified for this condition. Mlynarek et al. ${ }^{55}$ formulated the same conclusion resulting the use of PRP as effective as conventional treatment for Achilles tendinopathy.

Our retrospective study combines two regenerative treatments (ESWT or PRP injections) with a posttreatment eccentric exercise protocol. The results regarding both treatment groups are interesting. We note that the pain decreases considerably at first follow-up and this improvement is persistent at 4 and 6month in PRP injections group, whereas it decreases progressively at every time-point in ESWT group. Overall both treatments are effective on reduction pain and there is no significant difference of VAS score in between-analysis at all follow-up time points. Functional recovery and return to sport are been evaluated with VISA-A score. Data show that VISA-A score in PRP group increases progressively and significantly at every assessment, whereas in ESWT group a significant improvement was observed only at the first and second follow-up visits. Although our study is retrospective, we want to emphasize the interesting significant difference of VISA-A score at the time of the 4-month follow-up in favour of ESWT treatment.

Regarding treatment satisfaction, percentages of satisfied patient increase at every follow-up time point until $80 \%$ at third assessment and without significantIy differences between the two treatment groups. Moreover, regardless of treatment used, almost all the patients with a VISA-A score improvement greater than 30 points referred satisfaction.

In conclusion, our results allow us not only to confirm treatment with ESWT as a safe and effective treatment for the IAT, as previously confirmed by literature, but also to consider infiltrative treatment with $\mathrm{PRP}$ as a valuable conservative therapy for patients involved in physical activity with this disease.

This study has various limitations. The main one is the poor information about real patient compliance to post-treatment protocol and about complete recovery to sport. At 6-month follow-up, in fact, there were incomplete information concerning the sports management: all professional athletes had reported complete recovery training and competitions, while informations about amateur athletes in many cases have been insufficient.

Moreover, as the absence of complete information about compliance to post-treatment protocol, we aren't able to identify how much our satisfying results depend on eccentric loading. Furthermore, stratified sampling of patients to subgroups for retrocalcaneal bursitis, tendon calcification, and sports activity was not possible due to the study's small sample size.

Lastly, this work is a retrospective study with its intrinsic limitations. For example, there are no data about treatment response of retrocalcaneal bursitis and/or tendon calcification, coexisting with IAT, because it is not a parameter usually reported in our assessments.

\section{Conclusions}

Our study confirms that both ESWT and PRP injections are effective, safe and comparable in the treatment of insertional Achilles tendinopathy in physically active people. On the basis of our results, such as the evidence of satisfaction in $80 \%$ patients at the 6 month follow-up, we decided to continue to employ these two treatments at our clinical practice. Unfortunately, we were not able to define the most effective regenerative treatment for IAT, so further studies are warranted to establish a first-line treatment.

\section{Conflict of interest}

The Author has no financial or personal relationships with other people or organizations that could inappropriately influence their work.

\section{References}

1. Vulpiani MC, Guzzini M, Ferretti A. Operative treatment of chronic Achilles tendinopathy. Int Orthop. Epub 2003;27(5): 307-310.

2. Maffulli N, Via AG, Oliva F. Chronic Achilles Tendon Disorders: Tendinopathy and Chronic Rupture. Clin Sports Med. 2015 34(4):607-624.

3. Maffulli N, Sharma P, Luscombe KL. Achilles tendinopathy: aetiology and management. J R Soc Med. 2004;97(10):472476.

4. Maffulli N, Khan KM, Puddu G. Overuse tendon conditions: time to change a confusing terminology. Arthroscopy. 1998;14(8):840-843.

5. Maffulli N, Testa V, Capasso G, Sullo A. Calcific insertional Achilles tendinopathy: reattachment with bone anchors. Am J Sports Med. 2004;32(1):174-182.

6. Maffulli N, Wong J, Almekinders LC. Types and epidemiology of tendinopathy. Clin Sports Med. 2003;22(4):675-692.

7. Perugia L, Ippolito E, Postacchini F. A new approach to the pathology, clinical features and treatment of stress tendinopathy of the Achilles tendon. Ital J Orthop Traumatol. 1976;2 (1):5-21.

8. Puddu G, Ippolito E, Postacchini F. A classification of Achilles tendon disease. Am J Sports Med. 1976;4(4):145-150.

9. Clain MR, Baxter DE. Achilles tendinitis. Foot Ankle. 1992;13(8):482-487.

10. Del Buono A, Chan O, Maffulli N. Achilles tendon: functional anatomy and novel emerging models of imaging classification. Int Orthop. 2013;37(4):715-721.

11. Kvist M. Achilles tendon injuries in athletes. Sports Med. 1994;18(3):173-201

12. Järvinen M. Epidemiology of tendon injuries in sports. Clin Sports Med. 1992;11(3):493-504.

13. Leppilahti J, Orava S, Karpakka J, Takala T. Overuse injuries of the Achilles tendon. Ann Chir Gynaecol. 1991;80(2):202207.

14. Johansson C. Injuries in elite orienteers. Am J Sports Med. 1986;14(5):410-415.

15. Lysholm J, Wiklander J. Injuries in runners. Am J Sports Med. 1987;15(2):168-171.

16. Fahlström $\mathrm{M}$, Lorentzon $\mathrm{R}$, Alfredson $\mathrm{H}$. Painful conditions in the Achilles tendon region in elite badminton players. Knee Surg Sports Traumatol Arthrosc. Epub. 2002;10(1):57-60. 
17. Orava S, Leppilahti J, Karpakka J. Operative treatment of typical overuse injuries in sport. Ann Chir Gynaecol. 1991;80(2): 208-211.

18. Järvinen M. Lower leg overuse injuries in athletes. Knee Surg Sports Traumatol Arthrosc. 1993;1(2):126-130.

19. Schepsis AA, Jones $\mathrm{H}$, Haas AL. Achilles tendon disorders in athletes. Am J Sports Med. 2002;30(2):287-305.

20. Myerson M, McGarvey W. Disorders of the Achilles insertion and Achilles tendinitis. Instr Course Lect. 1999;48:211-218.

21. Notarnicola A, Maccagnano G, Tafuri S, Forcignanò MI, Panella A, Moretti B. CHELT therapy in the treatment of chronic insertional Achilles tendinopathy. Lasers Med Sci. 2014; 29(3):1217-25.

22. Khan KM, Cook JL, Kannus P, Maffulli N, Bonar SF. Time to abandon the "tendinitis" myth. BMJ. 2016;324(7338):626627.

23. Sharma $P$, Maffulli N. Tendon injury and tendinopathy: healing and repair. J Bone Joint Surg Am. 2005;87(1):187-202.

24. Wang JHC, losifidis MI, Fu FH. Biomechanical basis for tendinopathy. Clin Orthop Relat Res. 2006;443:320-332.

25. Benjamin M, Moriggl B, Brenner E, Emery P, McGonagle D, Redman S. The "enthesis organ" concept: why enthesopathies may not present as focal insertional disorders. Arthritis Rheum. 2004;50(10):3306-3313.

26. Jonsson P, Alfredson H, Sunding K, Fahlstro M, Cook J. New regimen for eccentric calf-muscle training in patients with chronic insertional Achilles tendinopathy: results of a pilot study. Br J Sports Med. 2008;42(9):746-749.

27. Alfredson $\mathrm{H}$, Lorentzon R. Chronic Achilles tendinosis: recommendations for treatment and prevention. Sports Med. 2000;29:135-146.

28. Almekinders LC. Tendinitis and other chronic tendinopathies. J Am Acad Orthop Surg. 1998;6(3):157-164.

29. Baxter DE, Zingas $C$. The foot in running. J Am Acad Orthop Surg. 1995;3(3):136-145.

30. McCrory JL, Martin DF, Lowery RB et al. Etiologic factors associated with Achilles tendinitis in runners. Med Sci Sports Exerc. 1999;31(10):1374-1381.

31. McGarvey WC, Palumbo RC, Baxter DE, Leibman BD. Insertional Achilles tendinosis: surgical treatment through a central tendon splitting approach. Foot Ankle Int. 2002;23(1):19-25

32. Nigg BM. The role of impact forces and foot pronation: a new paradigm. Clin J Sport Med. 2001;11(1):2-9.

33. Paavola M, Kannus P, Jarvinen TA, Khan K, Jozsa L, Jarvinen M. Achilles tendinopathy. J Bone Joint Surg Am. 2002;84A(11):2062-2076.

34. Saltzman CL, Tearse DS. Achilles tendon injuries. J Am Acad Orthop Surg. 1998;6(5):316-325.

35. Sorosky B, Press J, Plastaras C, Rittenburg J. The practical management of Achilles tendinopathy. Clin J Sport Med. 2004;14(1):40-44.

36. Furia JP. High-energy extracorporeal shock wave therapy as a treatment for insertional Achilles tendinopathy. Am J Sports Med. 2006;34(5):733-740.

37. IPG312 Extracorporeal shock-wave therapy for refractory Achilles tendinopathy: guidance. 2011.

38. Vulpiani MC, Trischitta D, Trovato P, Vetrano M, Ferretti A. Extracorporeal shockwave therapy (ESWT) in Achilles tendinopathy. A long-term follow-up observational study. J Sports Med Phys Fitness. 2009;49(2):171-176.
39. Rompe JD, Furia J, Maffulli N. Eccentric loading compared with shock-wave treatment for chronic insertional Achilles tendinopathy: a randomized controlled trial. J Bone Joint Surg Am. 2008;90(1):52-61.

40. Furia JP. Extracorporeal shockwave therapy in the treatment of chronic insertional Achilles tendinopathy. Orthopade. 2005;34(6):571-578.

41. Kearney RS, Parsons N, Costa ML. Achilles tendinopathy management: A pilot randomised controlled trial comparing platelet-richplasma injection with an eccentric loading programme. Bone Joint Res. 2013;17;2(10):227-232.

42. Ferrero G, Fabbro E, Orlandi D et al. Ultrasound-guided injection of platelet-rich plasma in chronic Achilles and patellar tendinopathy. J Ultrasound. 2012;8;15(4):260-266.

43. Sadoghi P, Rosso C, Valderrabano V, Leithner A, Vavken P. The role of platelets in the treatment of Achilles tendon injuries. J Orthop Res. 2013;31(1):111-118.

44. Visco V, Vulpiani MC, Torrisi MR, Ferretti A, Pavan A and Vetrano M. Experimental studies on the biological effects of extracorporeal shock wave therapy on tendon models. A review of the literature. Muscles, ligaments and tendons journal. 2014;4:357-361.

45. Zhou Y, Wang J H-C. PRP Treatment Efficacy for Tendinopathy: A Review of Basic Science Studies. Biomed Res Int 2016;2016:9103792.

46. Wiegerinck JI, Kerkhoffs GM, van Sterkenburg MN, Sierevelt IN, van Dijk CN. Treatment for insertional Achilles tendinopathy: a systematic review. Knee Surg Sports Traumatol Arthrosc. 2013;21(6):1345-1355.

47. Padulo J, Oliva F, Frizziero A, Maffulli N. Muscles, Ligaments and Tendons Journal - Basic principles and recommendations in clinical and field Science Research: 2016 Update. MLTJ. 2016;6(1):1-5.

48. Robinson JM, Cook JL, Purdam C et al. The VISA-A questionnaire: a valid and reliable index of the clinical severity of Achilles tendinopathy. Br J Sports Med. 2001;35(5):335-341.

49. Maffulli N, Longo UG, Testa V, Oliva F, Capasso G, Denaro V. Italian translation of the VISA-A score for tendinopathy of the main body of the Achilles tendon. Disabil Rehabil. 2008;30(2022):1635-1639.

50. Kremer E, Atkinson JH, Ignelzi RJ. Measurement of pain: patient preference does not confound pain measurement. Pain. 1981;10(2):241-248.

51. Roles NC, Maudsley RH. Radial tunnel syndrome: resistant tennis elbow as a nerve entrapment. J Bone Joint Surg Br. 1972;54(3):499-508

52. Taylor J, Dunkerley S, Silver D, et al. Extracorporeal shockwave therapy (ESWT) for refractory Achilles tendinopathy: A prospective audit with 2-year follow up. Foot (Edinb). 2016 26:23-29.

53. Di Matteo B, Filardo G, Kon E, Marcacci M. Platelet-rich plasma: evidence for the treatment of patellar and Achilles tendinopathy-a systematic review. Musculoskelet Surg. 2015;99(1):1-9.

54. Filardo G, Di Matteo B, Kon E, Merli G, Marcacci M. Plateletrich plasma in tendon-related disorders: results and indications. Knee Surg Sports Traumatol Arthrosc. 2016.

55. Mlynarek RA, Kuhn AW, Bedi A. Platelet-Rich Plasma (PRP) in Orthopedic Sports Medicine. Am J Orthop (Belle Mead NJ). 2016;45(5):290-326. 